\title{
Investigation of Factors Influencing on the Autoclave Tests Re- sults of Internal Anticorrosive Polymer Coatings
}

\author{
Mark Kovalev ${ }^{1,}{ }^{*}$, Ekaterina Alekseeva ${ }^{1}$, Nikita Shaposhnikov ${ }^{1}$ and Daniil Lyashenko ${ }^{1}$ \\ 1 Affiliation 1; Peter the Great St.Petersburg Polytechnic University (SPbPU), 195251, Russia, St.Petersburg, \\ Polytechnicheskaya, 29 \\ * Correspondence: kovalev.ma@spbstu.ru
}

Citation: Kovalev, M.; Alekseeva, E.; Shaposhnikov, N.; Lyashenko, D. Investigation of Factors Influencing on the Autoclave Tests Results of Internal Anticorrosive Polymer Coatings. 2021, volume number, $\mathrm{x}$.

https://doi.org/10.3390/xxxxx

Published: 22 February 2021

Publisher's Note: MDPI stays neutral with regard to jurisdictional claims in published maps and institutional affiliations.

\section{Copyright: (c) 2021 by the authors.} Submitted for possible open access publication under the terms and conditions of the Creative Commons Attribution (CC BY) license (http://creativecommons.org/licenses/by/4.0/).

\begin{abstract}
The use of polymer coatings is one of the most common methods of protecting metal structures from corrosion damage. For example, in the oil and gas industry, polymer coatings are used to protect the inner surfaces of oilfield pipelines. One of the open questions is predicting the life of the selected protective coating. Existing research methods are aimed at assessing the quality of coating application and its behavior under conditions that simulate operational ones, but they do not allow to talk about a guaranteed service life. In this regard, laboratory tests should include tests as close as possible to operational influences in order to identify the deficiencies of the investigated coatings. The paper discusses the methodological features of autoclave testing with a pressure drop for polymer powder coatings. Autoclave tests simulate operating conditions, but the way they are performed can affect the final result. Autoclave decompression tests were carried out in environments containing $\mathrm{CO}_{2}$ and $\mathrm{H}_{2} \mathrm{~S}$ gases. The effects of pressure release rate, temperature and coating quality were evaluated. Methods for assessing the degradation of coatings after testing are considered. The result of the work is a description of the factors that can affect the results of autoclave decompression tests of polymer anticorrosive coatings.
\end{abstract}

Keywords: polymer coatings; autoclave testing; oil and gas; anticorrosion coatings; polymer coating testing

\section{Introduction}

In the oil and gas industry, corrosion is one of the main causes of equipment failure. Most of the infrastructure for production and transportation is made up of pipelines, so there are many solutions to protect them from corrosion damage [1]. One of the methods to protect the inner wall of pipes is to apply a protective coating. Various types of coatings are used in their composition [2], this article discusses polymer powder coatings.

Polymer powder coatings have been used in the oil and gas industry for more than 30 years [3], but the issues related to the assessment of their durability under expected operating conditions remain relevant [4]. There is an extensive list of tests to determine the quality of polymer coatings and resistance to aggressive conditions. As a rule, tests consist of exposure of coated samples in aggressive solutions at elevated temperatures and climatic tests. The disadvantage of this type of research is their duration. For example, when developing a new paint system, a 1000-hour test will significantly lengthen the development cycle. Autoclave test is one of the options for accelerating research, it combined effect of temperature, pressure and an aggressive environment

Autoclave testing is used to assess the resistance of both metals and coatings [5,6]. There are standards and recommendations for conducting such tests, but as a rule, such documents describe general steps for conducting $[7,8]$. When they are implemented, each specific laboratory may have its own methodological features of research. In the case of coating testing, two types of autoclave tests are used. These are tests for resistance to decompression release and immersion autoclave test followed by slow release of pressure. 
A decompression autoclave test is a simulation of an operational emergency, but also, due to the harsh test conditions, this test allows you to quickly assess the resistance of coatings and the quality of their application. Typically, the duration of such tests is 24 hours. This article discusses the features of such tests.

When planning autoclave decompression testing of coatings, special consideration should be given to the test operating temperature. When choosing it, it is necessary to take into account both the operating conditions and the specifications for the selected coating. As mentioned above, the methodology for conducting autoclave tests in different laboratories may differ. The article discusses issues such as repeatability of test results, assessment of coating quality using a 24-hour autoclave test, the effect of pressure release rate, and methods for assessing coating degradation after autoclave testing.

\section{Materials and Methods}

The studies were carried out on samples of $100 \times 50 \mathrm{~mm}$ in size with a polymer coating applied to them. Samples were made both from finished products (tubular products) and from plates prepared for laboratory research. Examples of samples are shown in Figure 1.

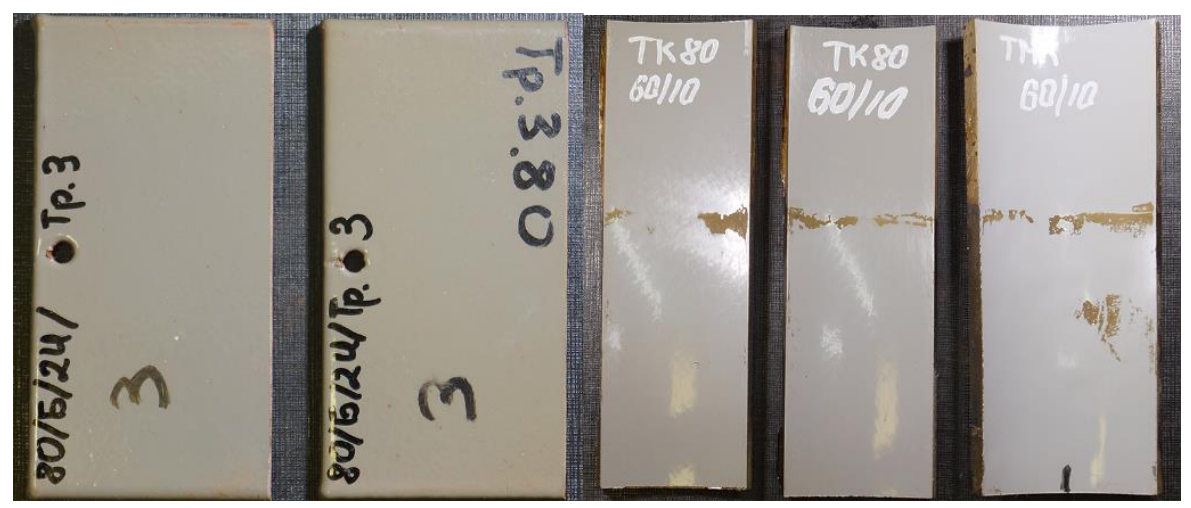

Figure 1. Testing samples

The tests were carried out in autoclaves with a volume of 1.5 and 3 liters. The smaller autoclave is made of stainless steel and was used for carbon dioxide research. The autoclave with a volume of 3 liters is made of titanium alloy and was used for testing with hydrogen sulfide. To provide pressure relief in less than 5 seconds, the outlet was larger in diameter than standard fittings. Autoclaves are shown in Figure 2.

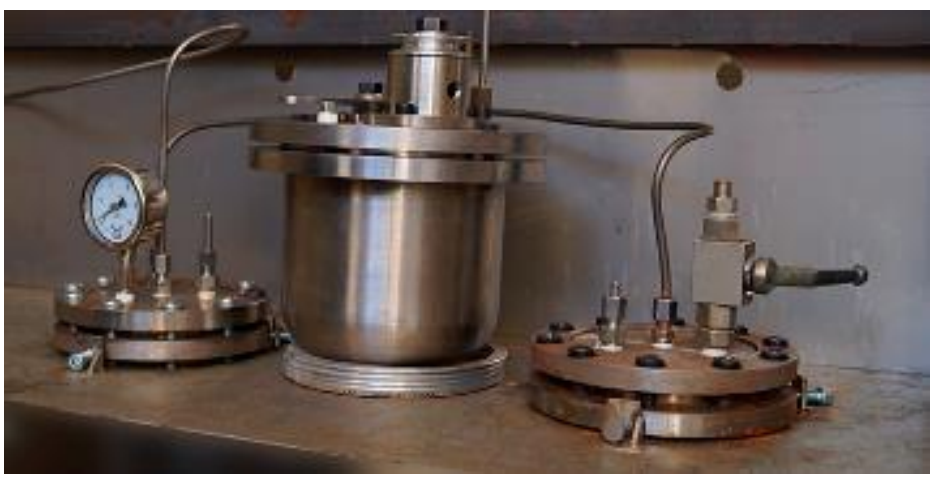

Figure 2. Autoclaves used for tests

A $5 \% \mathrm{NaCl}$ solution was used as a liquid medium. Carbon dioxide and hydrogen sulfide were used to saturate the autoclave capacity and create pressure. The autoclave was heated using a heating tape. The temperature was controlled by thermocouples. The autoclave test procedure included the following steps: the samples were placed in a $5 \%$ $\mathrm{NaCl}$ solution; a pressure of $0.5 \mathrm{MPa}$ was created with nitrogen; the pressure was maintained for 1 minute after which the pressure was released, the procedure was repeated 3 
times; at a temperature of $+20 \pm 5^{\circ} \mathrm{C}$, the autoclave was saturated with carbon dioxide until a pressure of $5 \mathrm{MPa}$ was reached and held for 1 hour until an equilibrium state was reached. The duration of the tests was 24 hours. At the end of the test, the pressure was released in less than 5 seconds.

The value was estimated using a pull of test using a tensile testing machine. The load increase rate did not exceed $1 \mathrm{MPa}$ per second, the total test duration did not exceed 90 seconds. For gluing the dollies, used 3M Scotch-Weld DP460 glue, the exposure time after gluing was 24 hours.

\section{Results}

Since carrying out autoclave tests is a process with its own methodological features, the first factor considered in the research process was the factor of repeatability of the results. Figure 3 shows samples after autoclave decompression tests under the same conditions, but tested in two autoclaves of the same design.

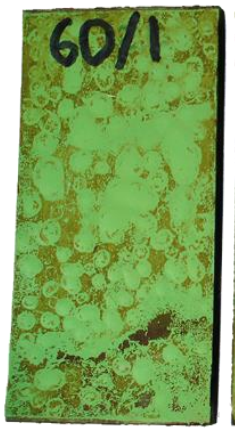

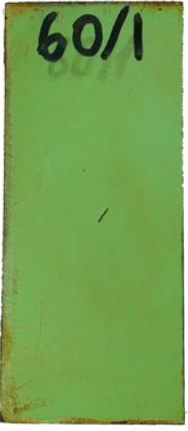

(a)

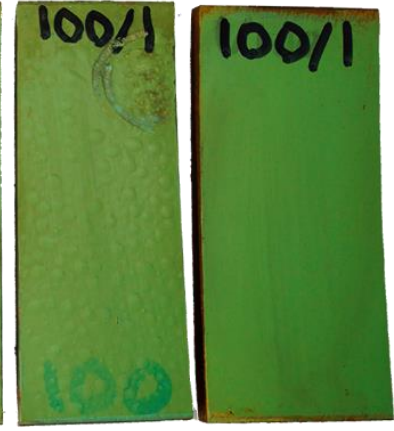

(b)

Figure 3. Samples after autoclave decompression tests: a) samples tested at a temperature $+60^{\circ} \mathrm{C}$; b) samples tested at a temperature $+90^{\circ} \mathrm{C}$.

As can be seen from Figure 3, samples of the same coating differ in their appearance after tests carried out under the same conditions. At the same time, their differences are critical for the results of assessing the resistance of coatings, since the presence of blisters indicates the instability of the system. Differences of this kind in the test results can indicate either the heterogeneity of the quality of the coating, or the unequal effect on the samples during the test, as well as the methodological features during each autoclave test. Inhomogeneity or poor coating performance in general can in most cases be assessed with a 24-hour autoclave decompression test. Figure 4 shows samples of the same polymer coating applied by different suppliers of pipe products.

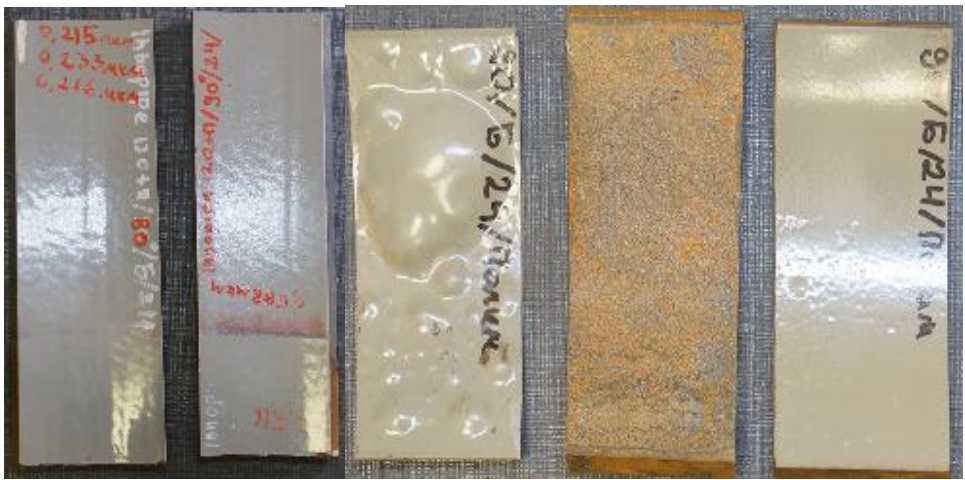

(a)

(b)

Figure 4. Samples of the same coating from different manufacturers of products after autoclave decompression tests: a) samples from manufacturer $1 ; b)$ samples from the manufacturer 2 . 
Figure 4 shows that, after autoclave tests, coatings from manufacturer 2 either completely peeled off the coating from the substrate, or large-diameter bulges formed. This case demonstrates one of the distinct advantages of the autoclave decompression test, as the quality of the coating can be determined in 24 hours.

The effect of exposure time was also tested during coating studies. Figure 5 shows samples of coatings after autoclave decompression tests under the same conditions for one and three days.

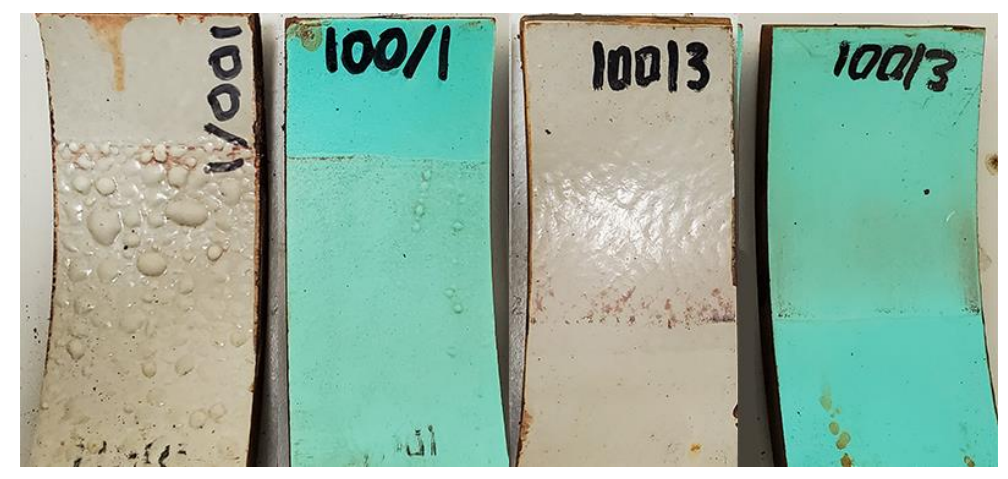

(a)

(b)

Figure 5. Samples after autoclave decompression tests with different duration of exposure under the same conditions: a) exposure for 24 hours; b) exposure for 72 hours.

Based on Figure 5, differences are seen in the state of the surface of the samples after 24 hours of exposure and holding in an autoclave for 72 hours. There are no blisters on the samples after 3 days of testing.

Since a decompression test consists of a sudden release of pressure at the end of the test, it is also necessary to understand the effect of the release time on test results. Typically, the pressure is released in less than 5 seconds. Figure 6 shows samples after autoclave decompression tests with different pressure release times within 5 seconds.
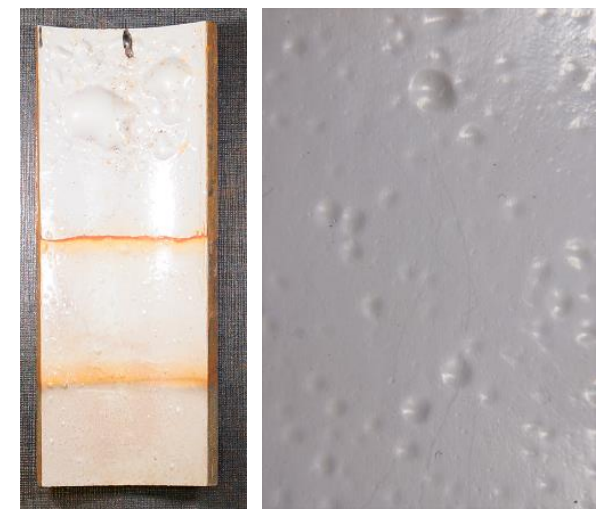

(a)
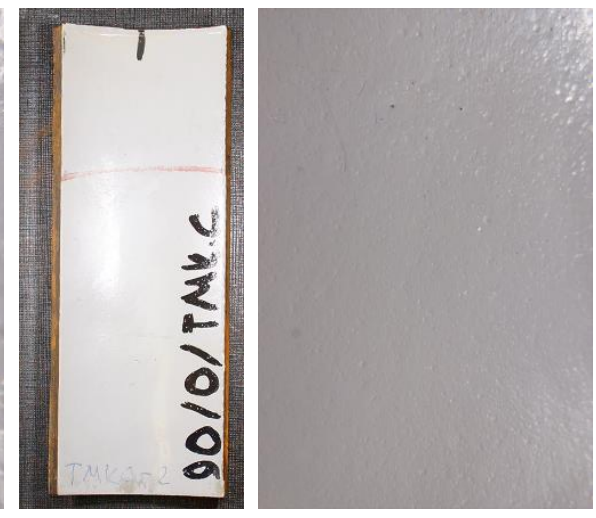

(b)

Figure 6. Influence of pressure release time during autoclave tests on decompression within 5 seconds: a) pressure release within 1.5 seconds; b) pressure relief in 4.9 seconds.

It can be seen from Figure 6 that during the discharge, blistering occurred in 1.5 seconds, and during the discharge close to 5 seconds, only a change in the coating roughness occurred.

The key issue in autoclave testing is the interpretation of the results. In some cases, the assessment is carried out only visually, and in some, the amount of adhesion of the coating is determined. Figure 7 shows the changes in the condition of the coating after autoclave-decompression tests at various temperatures. Samples were tested in the temperature range from $60^{\circ} \mathrm{C}$ to $130^{\circ} \mathrm{C}$ in $10^{\circ} \mathrm{C}$ steps 


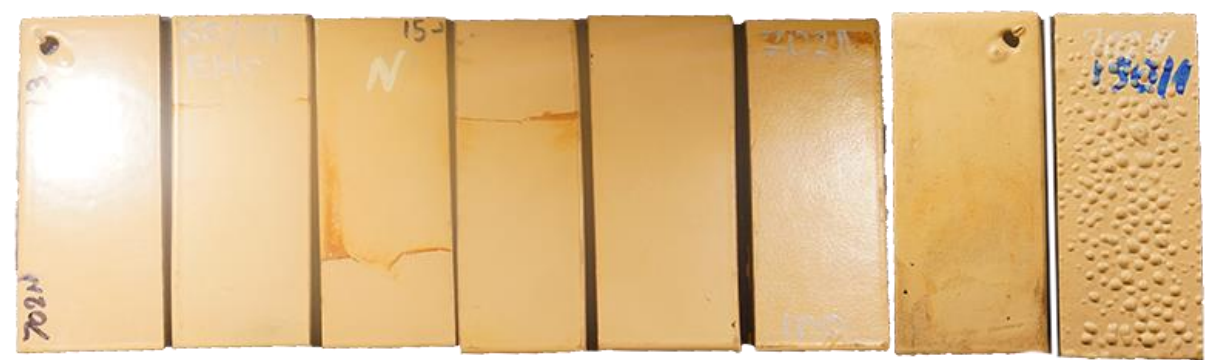

Figure 7. Change in the surface state of coated specimens after autoclave decompression tests as the test temperature increases

Figure 7 shows a gradual change in the state of the coating surface. With an increase in the temperature of autoclave tests, an increase in roughness occurs, which subsequently turns into the formation of blisters. In the case of examining the surface using optical microscopy, the following can be seen (Figure 8).

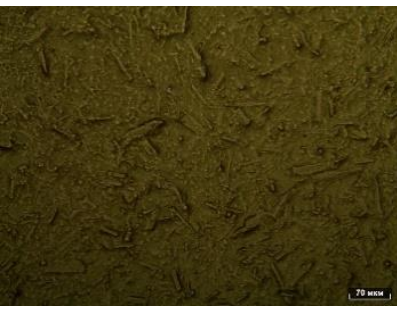

(a)

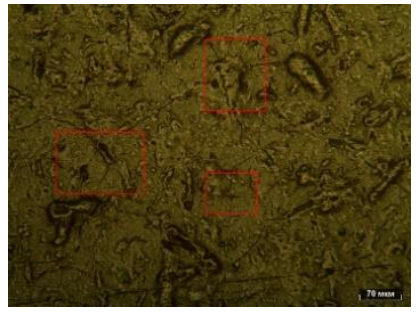

(b)

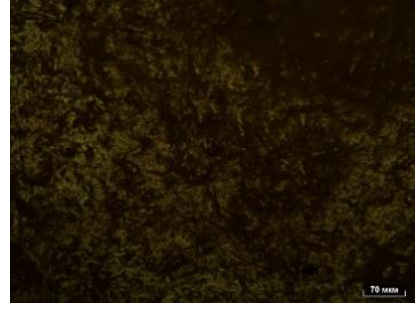

(c)

Figure 7. The change in the state of the surface of the coated samples after autoclave decompression tests as the test temperature increases, investigated with an increase of x100: a) witness sample; b) a sample tested at $+70^{\circ} \mathrm{C}$; c) a sample tested at $+90^{\circ} \mathrm{C}$;

As can be seen from Figure 7 , already at $+70^{\circ} \mathrm{C}$, the coating surface changes and microcracks form. At $+90^{\circ} \mathrm{C}$, the degradation of the coating becomes more pronounced, but if the test results are assessed by visual inspection, visible changes are only noticeable at temperatures of $+150^{\circ} \mathrm{C}$.

One of the most indicative methods for assessing the degradation of the coating is to determine the value of adhesion by the pull of test. The method allows one to obtain a numerical value, however, it also has its drawbacks when evaluating coatings with a coating adhesion value above $15 \mathrm{MPa}$. Table 1 shows the results of evaluating the value of adhesion before and after autoclave decompression tests.

Table 1. Results of pull of test after autoclave decompression test

\begin{tabular}{ccccc}
\hline Sample № & Value 1, MPa & Value 2, MPa & Value 3, MPa & Average value \\
\hline 1 & \multicolumn{5}{c}{ Samples before testing } \\
2 & 10,18 & 17 & 15 \\
3 & 18 & 21 & 19 & 19 \\
\hline 1 & 19 & 14 & 19 & 17 \\
2 & 15 & Samples after testing & 16 \\
3 & 15 & 15 & 16 & 17 \\
\end{tabular}

The results shown in table 1 can be called the optimal scenario when assessing the value of adhesion. Although there is a range of single values, the average adhesion values indicate no apparent degradation of the coating after autoclave testing. Table 2 shows the results of evaluating the value of adhesion of another coating after similar autoclave decompression tests. 
Table 2. Results of pull of test after autoclave decompression test

\begin{tabular}{ccccc}
\hline Sample № & Value 1, MPa & Value 2, MPa & Value 3, MPa & Average value \\
\hline 1 & \multicolumn{4}{c}{ Samples after testing } \\
2 & 17 & 8,5 & 25,4 & 17 \\
3 & 10,1 & 16,9 & 8,2 & 12 \\
\end{tabular}

As can be seen from Table 2 above, when determining the value of adhesion, there may also be a significant scatter of values, which complicates the interpretation of the test results and conclusions on the degradation of the coating after autoclave tests. In some cases, the criterion for the durability of the coating is the absence of a decrease in the adhesion value by more than $30 \%$. Due to the low convergence of the results according to this criterion, coatings that, in practice, would be stable under the selected operating conditions, can be rejected. When determining the value of adhesion by the pull-off method, it is necessary to pay attention to the nature of the pull-off; in some cases, the interpretation of the results is unambiguous. Figure 8 shows the appearance of the mold detachment zones for coating samples before and after autoclave tests.
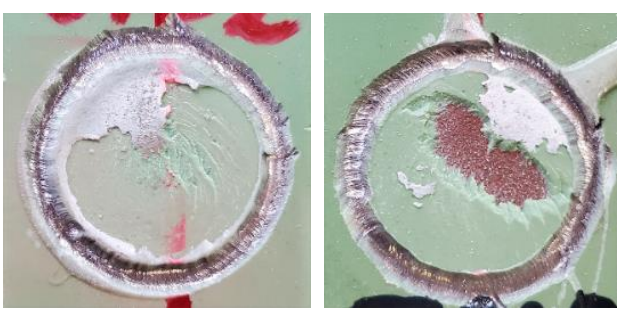

(a)
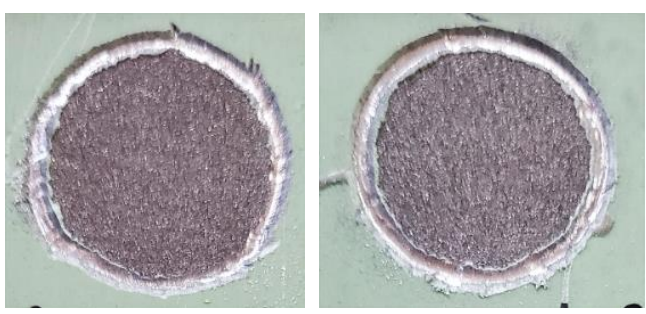

(b)

Figure 8. The nature of destruction in the zone after pull-off test: a) witnesses samples b) samples after testing

In Figure 8, the specimens after autoclave tests were completely detached from the metal substrate, which indicates the degradation of the coating. At the same time, in the witness samples, destruction was predominantly of a cohesive character between the coating layers. As an additional assessment method, coatings were examined after autoclave testing under an optical microscope. Metallographic sections were made in the transverse direction to study the coating structure before and after testing. Figure 9 shows the structures of the coatings.

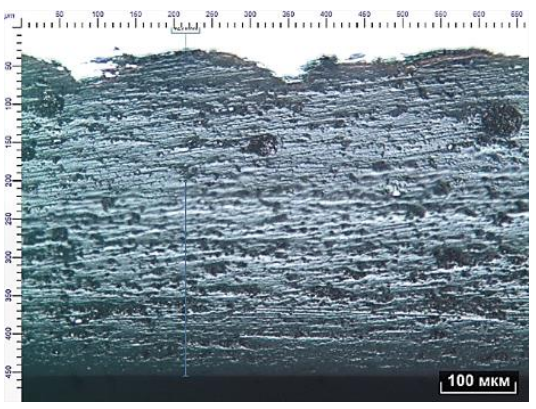

(a)

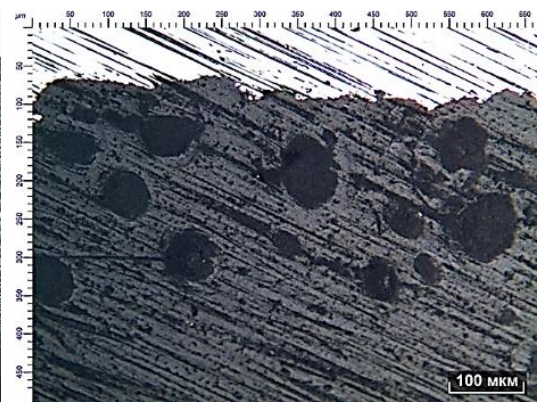

(b)

Figure 9. Coating structure after autoclave tests: a) sample before testing, $\times 100$; b) sample after testing, $\times 100$

Figure 9 shows that in the structure of the coating after autoclave decompression tests there were visible changes in the form of pore formation. This structural feature was absent on the sample before the tests. 


\section{Conclusions}

Based on the results of the conducted studies of the behavior of polymer powder coatings after autoclave decompression tests, methodological features were identified that must be paid attention to when conducting such studies. Autoclave testing allows you to assess the quality of the coating, however, during the study, it is worth testing at least 5 samples both to assess the repeatability of the results and to assess the quality of application to the pipe body. The pressure release time can affect the final test result and must be evaluated during the test. It was also noted that the absence of visible changes in the state of the coating after testing does not indicate the absence of degradation. As an additional assessment method, it is possible to use optical microscopy methods to compare the state of the coating before and after testing on the structure of the coating.

Funding: The research is partially funded by the Ministry of Science and Higher Education of the Russian Federation as part of World-class Research Center program: Advanced Digital Technologies (contract No. 075-15-2020-934 dated от 17.11.2020)

\section{References}

1. Popoola, L. T., Grema, A. S., Latinwo, G. K., Gutti, B., \& Balogun, A. S. (2013). Corrosion problems during oil and gas production and its mitigation. International Journal of Industrial Chemistry, 4(1), 1-15.

2. Ermakov, B. S., Alkhimenko, A. A., Shaposhnikov, N. O., Ermakov, S. B., Shatsky, T. E., \& Igolkin, A. F. (2020, April). The use of sprayed powders to create coatings in the welds of oilfield pipelines. In IOP Conference Series: Materials Science and Engineering (Vol. 826, No. 1, p. 012008).

3. Papavinasam, S. (2013). Corrosion control in the oil and gas industry. Elsevier.

4. Yudin, P., Petrov, S., Maximuk, A., \& Knyazeva, Z. (2019). Destruction mechanisms and methods of laboratory autoclave tests of internal coatings of oil pipes. In E3S Web of Conferences (Vol. 121, p. 01009).

5. Khar'Kov, A. A., Shakhmatov, A. V., Gyulikhandanov, E. L., \& Alekseeva, E. L. (2019). Comparative analysis of corrosionresistant alloys inconel 718 and ÉP718. Chemical and Petroleum Engineering, 54(9), 771-778.

6. Crolet, J. L., \& Bonis, M. R. (2000). How to pressurize autoclaves for corrosion testing under carbon dioxide and hydrogen sulfide pressure. Corrosion, 56(2), 167-182.

7. TM0185-2006-SG, Evaluation of Internal Plastic Coatings for Corrosion Control of Tubular Goods by Autoclave Testing

8. API RP 5L2. Recommended Practice for Internal Coating of Line Pipe for Non-corrosive Gas Transmission Service 